REVIEW ARTICLE

\title{
Biological factors that impinge on Chagas disease drug development
}

\author{
AMANDA F. FRANCISCO, SHIROMANI JAYAWARDHANA, MICHAEL D. LEWIS, \\ MARTIN C. TAYLOR and JOHN M. KELLY*
}

Department of Pathogen Molecular Biology, London School of Hygiene and Tropical Medicine, Keppel Street, London WC1E 7HT, UK

(Received 26 May 2017; revised 11 fuly 2017; accepted 14 fuly 2017; first published online 23 August 2017)

\begin{abstract}
SUMMARY
Chagas disease is caused by infection with the insect-transmitted protozoan Trypanosoma cruzi, and is the most important parasitic infection in Latin America. The current drugs, benznidazole and nifurtimox, are characterized by limited efficacy and toxic side-effects, and treatment failures are frequently observed. The urgent need for new therapeutic approaches is being met by a combined effort from the academic and commercial sectors, together with major input from not-for-profit drug development consortia. With the disappointing outcomes of recent clinical trials against chronic Chagas disease, it has become clear that an incomplete understanding of parasite biology and disease pathogenesis is impacting negatively on the development of more effective drugs. In addition, technical issues, including difficulties in establishing parasitological cure in both human patients and animal models, have greatly complicated the assessment of drug efficacy. Here, we outline the major questions that need to be addressed and discuss technical innovations that can be exploited to accelerate the drug development pipeline.
\end{abstract}

Key words: Chagas disease, Trypanosoma cruzi, drug development, disease pathogenesis.

\section{INTRODUCTION}

Five to eight million people in Latin America are infected with the protozoan parasite Trypanosoma cruzi, the aetiologic agent of Chagas disease (Hashimoto and Yoshioka, 2012; Bern, 2015). Infections are spread primarily by blood-sucking triatomine bugs, although other means of transmission include the congenital route, contaminated food and drink, organ transplantation and blood transfusion. Chagas disease is also becoming a global public health problem, with significant numbers of symptomatic cases now being detected within migrant populations, particularly in the USA and Europe, where the estimates of those infected are 300000 and 100000 , respectively (Bern et al. 2011; Pérez-Molina et al. 2012; Requena-Méndez et al. 2015).

Chagas disease has been divided into three discrete phases. The initial 'acute' stage, which occurs in the first 4-6 weeks post-infection, usually manifests as a mild and transient febrile condition, and in many cases, is asymptomatic. However, in children it can be more severe and sometimes fatal. With the development of a vigorous adaptive

* Corresponding author: Department of Pathogen Molecular Biology, London School of Hygiene and Tropical Medicine, Keppel Street, London WC1E 7HT, UK. E-mail: john.kelly@1shtm.ac.uk immune response, in which $\mathrm{CD}^{+} \mathrm{IFN}^{-} \gamma^{+} \mathrm{T}$ cells play a major role (Cardillo et al. 2015; Tarleton, 2015), the infection is suppressed, but sterile immunity is not achieved. This 'indeterminate' or 'asymptomatic chronic' stage is characterized by an intermittent and extremely low-level parasitaemia. However, $\sim 30 \%$ of infected individuals eventually proceed to the 'symptomatic chronic' stage, often decades after the primary infection. Cardiomyopathy develops in the majority of these individuals, whilst a minority (approximately $10 \%$ of those infected) suffer digestive tract megasyndromes (Ribeiro et al. 2012; Cunha-Neto and Chevillard, 2014). Chagas disease is a major cause of premature death in many areas of South America.

The front-line drugs used to treat T. cruzi infections are the nitroheterocyclic compounds benznidazole and nifurtimox (Wilkinson and Kelly, 2009; Gaspar et al. 2015). Both have been in use for almost 50 years, despite widespread evidence of treatment failures (Molina et al. 2014; Morillo et al. 2015, 2017). Other drawbacks include the long treatment period (often $60-90$ days), the frequency and severity of side-effects, and the potential for cross-resistance, which arises from the requirement of these nitroheterocyclic agents to be activated by the same parasite mitochondrial nitroreductase, TcNTR-1 (Wilkinson et al. 2008; Mejia et al. 2012). Although benznidazole has proven to be

Parasitology (2017), 144, 1871-1880. C Cambridge University Press 2017. This is an Open Access article, distributed under the terms of the CreativeCommons Attribution licence (http://creativecommons.org/licenses/by/4.0/), which permits unrestricted re-use, distribution, and reproduction in any medium, provided the original work is properly cited. 
effective at curing some acute and chronic T. cruzi infections, the extent to which it can prevent or alleviate chronic cardiac pathology remains uncertain (Molina-Berríos et al. 2013; Villar et al. 2014; Gruendling et al. 2015; Morillo et al. 2015). The only new compound recently advanced into clinical trials has been the anti-fungal agent posaconazole, which blocks ergosterol biosynthesis through inhi-

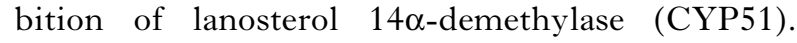
Unfortunately, posaconazole proved to have limited efficacy against chronic infections (Molina et al. 2014; Francisco et al. 2015; Morillo et al. 2017), despite some initially promising outcomes in experimental animal models (Molina et al. 2000; Ferraz et al. 2007).

The urgent need to develop more effective therapy against Chagas disease is now being tackled by large international, multidisciplinary teams (Katsuno et al. 2015; Chatelain, 2016). These have introduced a more systematic framework to drug development by bringing together expertise from both the academic and commercial sectors. However, it is clear that progress is being limited by gaps in our knowledge of parasite biology and disease pathogenesis, and that further technical innovations are required to accelerate the pathway that stretches from lead compound optimization to pre-clinical testing. Below, we highlight these major biological questions and discuss various approaches that could help to streamline the drug development process.

DOES PARASITE DIVERSITY IMPACT ON DRUG EFFICACY?

Trypanosoma cruzi is a highly diverse species with genetic distances between major lineages greater than those between members of the Trypanosoma brucei species complex (Franzén et al. 2011). Our understanding of parasite taxonomy has been complicated further by evidence of genetic exchange and widespread detection of putative hybrid strains (Machado and Ayala, 2001; Brisse et al. 2003; Gaunt et al. 2003; Lewis et al. 2011). The geographical range of T. cruzi extends from southern Chile and Argentina, through Central America, into wide areas of the southern USA (Brenière et al. 2016). The parasite can be transmitted by more than 100 species of triatomine vector and is capable of infecting most, if not all, mammalian species that it encounters (Messenger et al. 2015). The taxonomic categorization of T. cruzi has been subject to long, and at times vigorous, debate. Currently, the species is divided into six discrete typing units (DTUs) designated TcI-TcVI (Zingales et al. 2012) (Fig. 1), although a seventh, Tcbat, has recently been proposed (Marcili et al. 2009; Pinto et al. 2012). TcI is the most geographically dispersed DTU, with a range stretching from the USA to
Argentina, and although the other lineages are more localized, there is considerable overlap (for review, Miles et al. 2009; Brenière et al. 2016). This extensive diversity has prompted speculation as to whether there are correlations between parasite lineage, host preference, disease pathology and drug sensitivity.

All six DTUs are capable of infecting humans (Fig. 1); overall $\mathrm{TcI}$ and $\mathrm{TcV}$ infections are the most commonly identified, although other lineages predominate in some specific endogenous areas, such as TcII in parts of Brazil. Despite some circumstantial data, there has been no unequivocal evidence of a causative link between parasite diversity and disease outcome in humans. For example, suggestions that the absence of gastrointestinal megasyndromes in Venezuela compared with Brazil might be associated with genetic differences in the populations of circulating parasites have yet to be validated (Messenger et al. 2015). There is substantial intralineage genetic diversity, particularly within TcI, II, III and IV (Lewis et al. 2011); however, this has rarely been taken into account, because in most studies, genotyping is only conducted at the lineage level. Studies using experimental animal models do show that there can be important differences in virulence between individual strains (Schlemper et al. 1983; Postan et al. 1987; Espinoza et al. 2010; Rodriguez et al. 2014; Lewis et al. 2016), although no candidate genetic factors have been identified. The increasing availability of genomic technologies should enable progress to be made in delineating the extent to which parasite genetics contributes to disease outcome.

There have been multiple reports of wide divergence in the drug susceptibility of different $T$. cruzi strains. In a survey which encompassed representatives of DTUs I-VI, significant differences in benznidazole sensitivity were identified, but there was no correlation with parasite lineage (Villarreal et al. 2004). These results were consistent with data obtained from a panel of 28 parasite isolates from Colombia, where in vitro $\mathrm{EC}_{50}$ values against benznidazole ranged from 1 to $35 \mu \mathrm{M}$ (Mejia et al. 2012). Again, there was no obvious correlation between sensitivity and lineage (the panel contained DTU I and II strains), or with the biological origin of the parasites, either insect vector, small mammal or human. This extensive natural variation in benznidazole sensitivity was independent of TcNTR-1 sequence, implying that it must be associated with additional factors. In another report, parasites belonging to each of the DTUs were tested in vitro against several nitroheterocylic drugs and other lead compounds (Moraes et al. 2014). Although parasite strains exhibited a range of susceptibilities to individual drugs (e.g. up to 8 -fold in the case of nifurtimox), there was no evidence that any of the lineages were intrinsically more resistant to drugs in general. In 


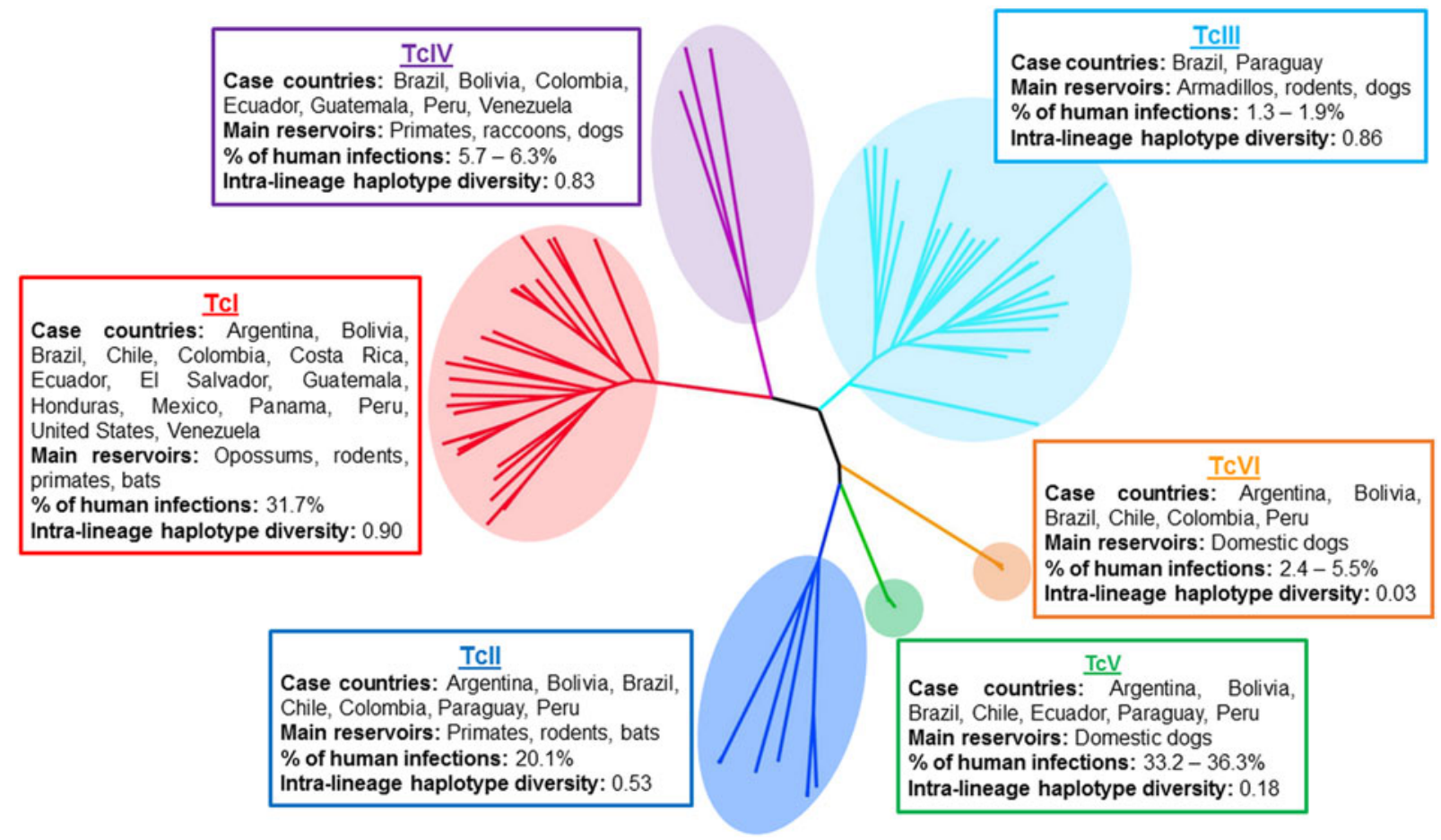

Fig. 1. Key features of the Trypanosoma cruzi major genetic lineages. The phylogenetic tree was reconstructed using multi-locus microsatellite genotype data, adapted from Lewis et al. (2011). Haplotype diversity is based on mitochondrial gene sequences for COII and ND1 reported in Lewis et al. (2011), and the values shown indicate the probability that two randomly selected haplotypes will be different. The percentage of human infections is estimated from metadata compiled by Brenière et al. (2016), which encompass all isolates derived from human infections $(n=1902)$ and typed to each lineage. These values may reflect historical variation in sampling intensities between endemic areas.

vivo studies have been inconsistent in terms of linking drug susceptibility to parasite lineage. For example, while Toledo et al. (2003) found that TcI-infected mice were less frequently cured by benznidazole or itraconazole than TcII- or TcVinfected mice, they also observed extensive heterogeneity in drug sensitivity between strains within these lineages. Similar intra-lineage variations were also reported when the curative potential of benznidazole was assessed with a number of Brazilian strains (Teston et al. 2013). In a study with the other front line drug nifurtimox, no association was found between therapeutic effectiveness and parasite lineage (Oliveira et al. 2017).

In summary, although natural T. cruzi isolates can display large variations in drug susceptibility, there is little evidence to link this with their taxonomic designation at the DTU level. As a general observation, intra-lineage differences seem to be as extensive as those between lineages. This highlights that assessment of the ability of lead compounds to display in vivo activity against a wide panel of isolates, reflecting the diverse phylogeny and geographical range of T. cruzi, must be considered an integral step in the drug development pathway. The importance of this is emphasized further by the commonality of mixed infections (Bontempi et al. 2016).
ARE ALL PARASITE LIFE-CYCLE STAGES EQUALLY SUSCEPTIBLE TO CHEMOTHERAPY?

From the drug development perspective, there are a number of important questions relating to the T. cruzi life-cycle that need to be addressed: (i) Is it necessary to kill all developmental forms to produce a curative outcome? (ii) Are all developmental forms equally susceptible to trypanocidal compounds? (iii) Is there a point in the life-cycle during chronic stage infections where the parasites enter a biochemically quiescent or dormant phase? The T. cruzi life-cycle involves a series of differentiation steps, in which the parasite passes through both replicative and non-replicative stages. In the classical text-book version of the mammalian lifecycle, which has been established for more than a century, insect-transmitted non-replicating metacyclic trypomastigotes invade host cells, differentiate into small round-shaped, non-flagellated intracellular amastigotes, and then divide by binary fission in the cytosol. When they reach a threshold level, which may be several hundred per infected cell, they differentiate into non-dividing flagellated trypomastigotes, which are released following lysis of the host cell. The trypomastigotes can then reinvade other cells, or be taken up in a bloodmeal by a feeding triatomine bug. 


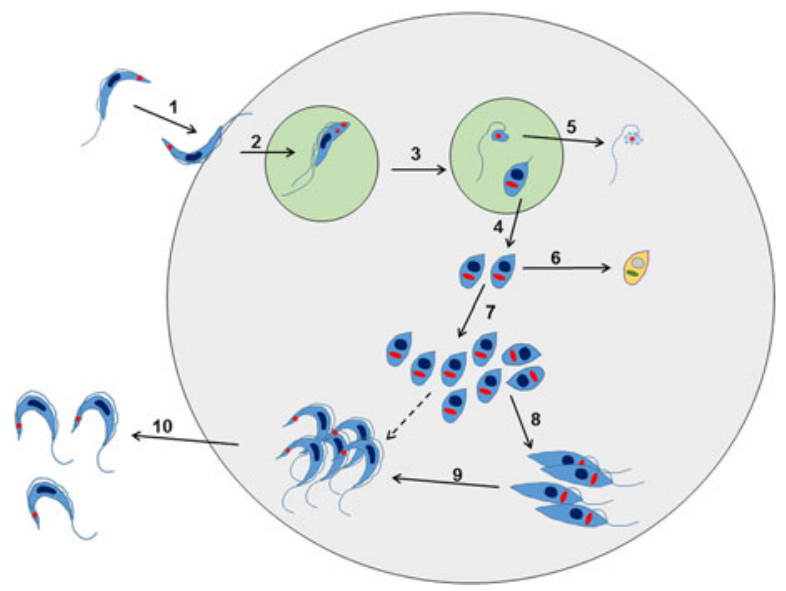

Fig. 2. Overview of the intracellular life cycle of Trypanosoma cruzi in the mammalian host. (1) The metacyclic trypomastigote binds to receptors on the host cell surface resulting in the parasite being taken up into a parasitophorous vacuole. This occurs regardless of whether or not the host cell is phagocytic. (2) The parasite undergoes an asymmetric cell division following replication of the kinetoplast (red circle) and flagellum, but not the nucleus (Kurup and Tarleton, 2014). (3) This results in one daughter cell being a replication competent amastigote with a short flagellum, and the other being a dysnuclear flagellated cytoplasmic fragment. (4) The amastigote escapes into the cytoplasm and begins replication by binary fission. (5) The remaining parasite component is degraded by the proteasome and its antigens are presented on the surface. (6) Some amastigotes may become metabolically quiescent, although this is yet to be proven. Such amastigotes could reside long term in chronically infected tissue. (7) The amastigotes continue to replicate. (8) Amastigotes differentiate into an intracellular epimastigote-like form. It is not clear whether this is an obligate stage, or if they can go straight from amastigotes to trypomastigotes (dashed arrow). (9) The parasites finally differentiate into the flagellated bloodstream trypomastigotes, lyse the host cell and escape into the bloodstream or tissue fluids (10).

Further research has revealed that this established view of the life-cycle is rather superficial and that in reality, the process is almost certainly more complex (Fig. 2). For example, evidence for an intracellular epimastigote-like stage has been intermittently reported (for review, Tyler and Engman, 2001), although it is unclear whether this enigmatic form is simply an intermediate in the amastigote to trypomastigote transition, or represents an obligate intracellular stage of the life-cycle, with a distinct role in vivo. Similarly, amastigote-like forms with short flagella, termed sphaeromastigotes, have also been widely reported, although these probably represent intermediate forms in the transition to epimastigotes, rather than distinct life-cycle stages (Tyler and Engman, 2001). Recently, trypomastigotes have also been shown to have the capacity to differentiate into an epimastigote-like morphological form, after transition through an amastigote-like intermediate. These recently differentiated epimastigotes (rdEpi) display a distinct proteomic fingerprint, are complement-resistant, able to invade phagocytic and cardiac cells (but not fibroblasts or epithelial cells), and can initiate an infection in mice (Kessler et al. 2017). It has also been demonstrated that the initial differentiation from the metacyclic trypomastigote involves an asymmetric cell division (Fig. 2), which results in one amastigote and one 'zoid' - a cell with a kinetoplast, but no nucleus. The zoid quickly dies and is degraded by the host cell machinery with some of its antigens being presented on the infected cell surface (Kurup and Tarleton, 2014). The role(s) of the intermediate in vivo forms are not well defined, and it is unclear whether their sensitivity (or otherwise) to test compounds is adequately captured using current in vitro screening systems. Efficacy testing against trypomastigotes is now routinely incorporated into drug screening protocols (Cortes et al. 2015; Guedes-da-Silva et al. 2016). However, the sensitivity of other intracellular and/or intermediate stages is intrinsically difficult to establish, and it is unknown whether it is necessary to target each of these morphological forms to eradicate an infection.

The importance of understanding the interplay between drug activity and the parasite life-cycle has been highlighted by studies with CYP51 inhibitors, such as posaconazole and ravuconazole. Although these drugs have low nanomolar $\mathrm{EC}_{50}$ values against a range of $T$. cruzi strains, they seem unable to completely clear parasites from infected mammalian cells in culture (Moraes et al. 2014). Consistent with this, studies in murine models have shown that although posaconazole is highly effective at reducing the parasite burden when administered at $10 \mathrm{mg} \mathrm{kg}^{-1} \mathrm{day}^{-1}$ for 25 days, it does not eliminate the infection, even when the dose is increased 10-fold, or when the treatment period extended to 40 days (Khare et al. 2015). One possibility is that treatment could induce resistance by promoting higher level expression of the lanosterol $14 \alpha$-demethylase target. Alternatively, there could be a sub-population of dormant, metabolically quiescent parasites within infected host cells, which have a reduced requirement for ergosterol biosynthesis. Addressing if this is the case, must be considered a major research goal, particularly because the existence of such parasites might have broader relevance for many other classes of drug.

In addition to the above, it could be that metabolically dormant parasites exist in some tissue niches during chronic infections. Investigating this is a major technical challenge, since parasites are present in extremely low numbers during the chronic stage. As outlined below, bioluminescence imaging allows parasites to be localized to specific organs and tissues in murine models, with the 


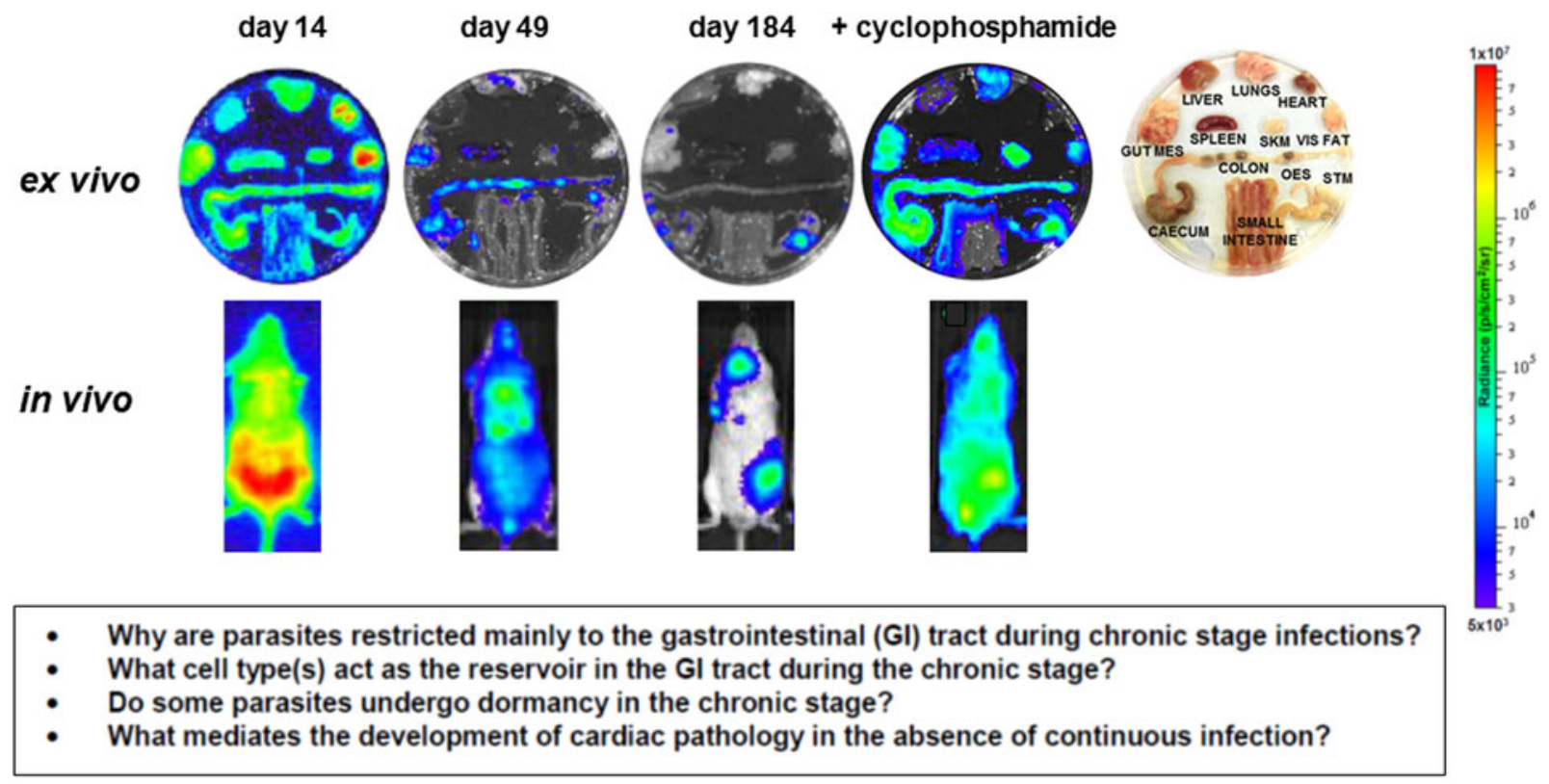

Fig. 3. Parasite tropism during Trypanosoma cruzi infections in a mouse model. BALB/c mice infected with bioluminescent T. cruzi strain CL Brener (Lewis et al. 2014) were imaged at various stages post-infection, as indicated. Upper; ex vivo imaging of organs/tissues removed from mice and soaked in D-luciferin. The identity of each organ/tissue is indicated (right). Lower; in vivo imaging of infected mice. The bioluminescence images on the right-hand side are of a chronically infected mouse which has been immunosuppressed by cyclophosphamide treatment (Lewis et al. 2014). Heatmaps are on $\log 10$ scales and indicate intensity of bioluminescence from low (blue) to high (red). Inset: summary of the major unanswered questions.

colon and/or stomach identified as the major reservoirs during the chronic stage (Fig. 3) (Lewis et al. 2014,2016). However, the technology is not sufficiently sensitive or applicable to allow the microscopic detection of individual infected cells. One strategy currently being developed involves the generation of parasites that express dual bioluminescence:fluorescence reporters, so that infected foci in tissues can be pinpointed, excised and sectioned, and then intracellular parasites visualized by fluorescence microscopy (Taylor et al. unpublished). This type of approach will be essential if the phenotype of the reservoir host cells is to be determined, the metabolic and replicative status of the residual parasites defined, and their response to trypanocidal drugs assessed.

DOES PARASITE TROPISM DURING CHRONIC INFECTIONS HAVE THERAPEUTIC IMPLICATIONS?

In humans, infections with $T$. cruzi are considered to be life-long (Álvarez et al. 2014; Cardoso et al. 2016). However, during the chronic phase, parasites are highly focal, present at extremely low levels, and only sporadically detectable in the bloodstream. This can limit the accurate diagnosis of on-going infections, even with PCR-based methodology (Schijman et al. 2011), and is an important complicating factor in clinical trials. By necessity, most studies on parasite tropism and persistence during human chronic infections have focused on tissue samples retrieved at autopsy, or following organ transplantation. The degree to which these findings are relevant to the majority of infected people is uncertain, and as a result, parasite tropism in asymptomatic individuals is poorly understood.

Intracellular amastigotes have been detected in some chagasic heart samples using histology (Benvenuti et al. 2014; Kransdorf et al. 2016), and more frequently using PCR or antibody-based techniques (Bellotti et al. 1996; Schijman et al. 2004; Burgos et al. 2010). Evidence from transplantationlinked transmission, in both endemic and nonendemic regions (Kun et al. 2009; Huprikar et al. 2013), suggests that parasites can be present in a number of organs, with infections more common after heart transplants, than those involving kidney or liver. Parasite DNA has also been detected in the oesophagus (Vago et al. 1996; Lages-Silva et al. 2001) and adipose tissue (Ferreira et al. 2011) during chronic infections, and parasites can become widely disseminated following reactivation of Chagas disease in immunosuppressed patients (see Fig. 3, as an example in a murine model), or those co-infected with HIV (for review, Lattes and Lasala, 2014). CNS involvement leading to meningoencephalitis is a common outcome in these situations (Cordova et al. 2008; Diazgranados, et al. 2009; Yasukawa et al. 2014).

Current knowledge on infection dynamics and parasite tropism during chronic T. cruzi infections 
in humans is insufficient to identify which tissue sites are important in terms of drug targeting and bioavailablity, or to determine if specific organs or tissues have a role in recrudescence. Given the practical difficulties in addressing these questions in infected patients, predictive experimental models have been at the forefront of the research effort. Animal models include dogs (Santos et al. 2016), primates (Vitelli-Avelar et al. 2017), chickens (Teixeira et al. 2011) and most commonly, mice, where several model systems are available which mimic aspects of disease pathology in humans (Eickhoff et al. 2010; Olivieri et al. 2010; Molina-Berríos et al. 2013; Sbaraglini et al. 2016). However, even in experimental models, difficulties in monitoring parasite burden and location during chronic infections have been a limiting step. With mice, these issues have been partially resolved by the development of highly sensitive bioluminescence imaging procedures, which for the first time enable chronic infections to be assessed in real time (Lewis et al. 2014, 2015). The system, which utilizes genetically modified parasites that express red-shifted luciferase (Branchini et al. 2010), has a limit of detection close to 100 parasites in inoculated mice and allows infections to be monitored in individual animals for more than a year (Lewis et al. 2014). In the BALB/c mouse - T. cruzi CL Brener (DTU VI) model, the infection peaks on day 14 post-inoculation (Fig. 3). Following induction of an adaptive immune response, the parasite burden then decreases by two to three orders of magnitude over the next 30-40 days, as the infection progresses to the chronic stage. Long-term infections are characterized by a dynamic profile, in which bioluminescence foci appear and disappear over a period of hours, in an apparently stochastic manner (Lewis et al. 2014). Similar patterns of infection occur with other parasite lineages and mouse strains, although with some differences in the precise timing of progression, presumably reflecting the influence of host and parasite genetics (Taylor et al. 2015; Lewis et al. 2016).

In the murine acute stage, the infection is pantropic, with parasites easily detectable by ex vivo imaging in all organs and tissues (Fig. 3). However, in the chronic stage, the colon and/or stomach are the primary sites of infection. Other organs, including the heart, are infected only sporadically, with the extent of this varying in different host:parasite strain combinations (Lewis et al. 2016). Myocarditis and heart fibrosis can develop in the absence of end-point cardiac infection, implying that the continuous presence of the parasite in the heart is not a pre-requisite for chagasic pathology. These data suggest a model where the gut is a permissive immunological niche that tolerates continuous low-level infection, with periodic trafficking of parasites, or more likely, parasite-infected cells, from this reservoir to other sites, including the heart. This leads to the generation of intermittent inflammatory immune responses that eliminate the transient infections in non-gut sites, but can result in collateral damage to surrounding tissue (Lewis and Kelly, 2016). It is implicit in this model that drug-mediated elimination of parasites from the gut reservoir sites should lead to parasitological cure in asymptomatic, immunocompetent individuals, with the immune system eradicating the remaining parasites from non-gut sites. While this model has yet to be proven, it does generate a number of questions with implications for drug development. First, what is the nature of the host cells in which parasites persist within the gut reservoir sites, and what is their immunological and metabolic status? Second, do parasites in these sites display dormancy, and if so, does this affect their sensitivity to therapeutic treatment? Third, do the transient bioluminescent foci in chronically infected mice represent infected cells undergoing trafficking, and what is their fate in an immunocompetent individual? Finally, can these insights into parasite tropism and persistence be extended from murine models to human patients?

The current inability to reliably cure chronic $T$. cruzi infections has led to speculation that the parasite might be able to survive in organs/tissues where drug access is limited. However, this appears not to be the case with nitroheterocyclic drugs, at least in mice. Treatment failures, in both acute and chronic infections, are not linked to a single or predominant site of recrudescence (Francisco et al. 2015, 2016). Consistent with this, a detailed study of benznidazole pharmacokinetics has revealed that inadequate bio-distribution is unlikely to be responsible for therapeutic failure during chronic phase murine infections (Perin et al. 2017). With posaconazole, the situation is less clear-cut. Although adipose tissue has been identified as a frequent site of cyclophosphamide-induced relapse after non-curative treatment of acute stage infections (Francisco et al. 2015), this was not observed in all mice. Adipose tissue has been implicated as a possible reservoir of recrudescence in other parasitic infections, including African trypanosomiasis (Trindade et al. 2016; Tanowitz et al. 2017); however, further work will be required before definitive conclusions can be drawn about the situation in T. cruzi.

DOES DRUG TREATMENT PREVENT OR ALLEVIATE CHRONIC DISEASE PATHOLOGY?

At a population level, the major health and economic burdens associated with Chagas disease result from chronic cardiac pathology. However, chronic stage studies, particularly on drug efficacy and disease pathogenesis, are made difficult by the scarce and highly focal nature of $T$. cruzi infection. As a result, the major research effort has focussed on the 
acute stage, where the monitoring of parasite load and the assessment of tissue tropism are more straightforward. There has been much discussion within the community on the underlying causes of chronic Chagas disease pathology (Gironès et al. 2005; Kierszenbaum, 2005; Gutierrez et al. 2009; Bonney and Engman, 2015). The context for this debate has been the inability to routinely detect parasites in the hearts of patients with cardiac damage and the non-specific (and sometimes autoreactive) polyclonal $\mathrm{B}$-cell and $\mathrm{T}$-cell responses that are characteristic of $T$. cruzi infection (Iwai et al. 2005; Bermejo et al. 2011). The central question has been: does chronic stage cardiac pathology develop as a consequence of autoimmunity, or does it result from parasite persistence and the generation of aberrant inflammatory responses within target organs? There is now a strong consensus that the presence of the parasite is a pre-requisite for heart pathology (for review, Bonney and Engman, 2015), although as mentioned above, the mode of cardiac infection may be one of episodic re-invasion rather than continuous persistence (Lewis and Kelly, 2016).

Cardiomyopathy develops in $20-30 \%$ of $T$. cruziinfected patients. Progressive heart failure, thromboembolism, ventricular arrhythmia, stroke and sudden death are common outcomes (Rossi et al. 2003; Carod-Artal, 2010; Carod-Artal and Gascon, 2010). Patient management is based on standard protocols for treating progressive cardiac failure (Ribeiro et al. 2012), despite the lack of robust randomized clinical trials to validate their use in cases of chagasic heart disease. The ability of drug treatment to prevent or alleviate the development of cardiac pathology is unresolved, and controversial. Despite this, the consensus view is that treatment should be offered to patients infected with $T$. cruzi, irrespective of their disease status. The validity of this approach is one of the central debates in the Chagas disease field. There is reasonable evidence from experimental models that curative treatment of acute stage infections results in reduced disease pathology in the longer term (Davies et al. 2010; Molina-Berríos et al. 2013; Assíria Fontes Martins et al. 2015; Gruendling et al. 2015). With chronic stage infections, the data are less clear-cut (Villar et al. 2014). For example, in a recent large multi-centre, randomized clinical trial, no significant improvements in terms of cardiomyopathy were observed 5 years after benznidazole treatment (BENEFIT trial; Morillo et al. 2015). However, because evidence of cardiomyopathy was a pre-requisite for enrolment in this trial, it has not been possible to draw conclusions about the type of outcomes that might be achievable by treating asymptomatic individuals.

Clinical trials to assess the link between antiparasitic treatment and reductions in pathology present numerous challenges. These include the long-term and diverse nature of the human disease, the toxicity of current drugs, the resulting compliance issues, and difficulties in demonstrating parasitological cure. This is complicated further by other variables such as the severity or otherwise of the acute stage infection, the possibility of re-infection/co-infection, host and parasite genetics, environmental factors and immune status. With advances in imaging technology, which allow realtime monitoring of chronic stage infections, it should be feasible to better exploit predictive animal models to investigate the rationale for using anti-parasitic drugs to prevent or alleviate symptomatic Chagas disease. Data from such experiments will be invaluable for informing the design of clinical trials aimed at establishing, for example, if there is a post-infection time limit within which curative therapy has to be administered to significantly impact on the development of cardiac pathology. The outcome of such studies should have major implications for the 5-8 million people infected with $T$. cruzi. However, it should be noted that currently, only $1 \%$ of those infected have access to diagnosis and treatment (DNDi, 2015).

\section{Concluding remarks}

The development of more effective drugs against Chagas disease is a major challenge for the biomedical research community. The complexity of the infection, combined with our limited understanding of parasite biology and disease pathogenesis are major factors that inhibit progress in this area. Addressing these problems will require a twin track strategy; basic research to address the questions outlined in this review, and applied research, with input from the not-for-profit consortia and the commercial sector, to exploit the resulting opportunities and fast-forward the drug development pipeline.

\section{FINANCIAL SUPPORT}

JMK acknowledges financial support from the British Heart Foundation (PG/13/88/30556), the Australian Research Council (LP140100560) and the Drugs for Neglected Diseases Initiative (DND $i$ ). MDL is supported by an EU Marie Curie Fellowship (625810).

\section{REFERENCES}

Álvarez, J. M., Fonseca, R., Borges da Silva, H., Marinho, C. R., Bortoluci, K. R., Sardinha, L. R., Epiphanio, S. and D'Império Lima, M. R. (2014). Chagas disease: still many unsolved issues. Mediators of Inflammation 2014, 912965

Assíria Fontes Martins, T., de Figueiredo Diniz, L., Mazzeti, A. L., da Silva do Nascimento, Á. F., Caldas, S., Caldas, I. S., de Andrade, I. M., Ribeiro, I. and Bahia, M. T. (2015). Benznidazole/itraconazole combination treatment enhances anti-Trypanosoma cruzi activity in experimental Chagas disease. PLoS ONE 10, e0128707.

Bellotti, G., Bocchi, E. A., de Moraes, A. V., Higuchi, M. L., BarberoMarcial, M., Sosa, E., Esteves-Filho, A., Kalil, R., Weiss, R., Jatene, A. and Pileggi, F. (1996). In vivo detection of Trypanosoma 
cruzi antigens in hearts of patients with chronic Chagas' heart disease. American Heart fournal 131, 301-307.

Benvenuti, L.A., Roggério, A., Nishiya, A.S., Campos, S. V., Fiorelli, A. I. and Levi, J.E. (2014). Trypanosoma cruzi persistence in the native heart is associated with high-grade myocarditis, but not with Chagas' disease reactivation after heart transplantation. The Fournal of Heart and Lung Transplantation 33, 698-703.

Bermejo, D. A., Amezcua Vesely, M. C., Khan, M., Acosta Rodríguez, E.V., Montes, C.L., Merino, M.C., Toellner, K. M., Mohr, E., Taylor, D., Cunningham, A.F. and Gruppi, A. (2011) Trypanosoma cruzi infection induces a massive extrafollicular and follicular splenic B-cell response which is a high source of non-parasite-specific antibodies. Immunology 132, 123-133.

Bern, C. (2015). Chagas' disease. New England Fournal of Medicine 373 456-466.

Bern, C., Kjos, S., Yabsley, M. J. and Montgomery, S. P. (2011). Trypanosoma cruzi and Chagas' disease in the United States. Clinical Microbiology Reviews 24, 655-681.

Bonney, K. M. and Engman, D. M. (2015) Autoimmune pathogenesis of Chagas heart disease: looking back, looking ahead. American fournal of Pathology 185, 1537-1547.

Bontempi, I. A., Bizai, M. L., Ortiz, S., Manattini, S., Fabbro, D., Solari, A. and Diez, C. (2016). Simple methodology to directly genotype Trypanosoma cruzi discrete typing units in single and mixed infections from human blood samples. Infection, Genetics and Evolution 43, 123-129. Branchini, B. R., Ablamsky, D. M., Davis, A.L., Southworth, T. L., Butler, B., Fan, F., Jathoul, A. P. and Pule, M. A. (2010). Red-emitting luciferases for bioluminescence reporter and imaging applications. Analytical Biochemistry 396, 290-297.

Brenière, S.F., Waleckx, E. and Barnabé, C. (2016) Over six thousand Trypanosoma cruzi strains classified into discrete typing units (DTUs): attempt at an inventory. PLoS Neglected Tropical Diseases 10, e0004792.

Brisse, S., Henriksson, J., Barnabé, C., Douzery, E. J., Berkvens, D., Serrano, M., De Carvalho, M. R., Buck, G. A., Dujardin, J. C. and Tibayrenc, M. (2003). Evidence for genetic exchange and hybridization in Trypanosoma cruzi based on nucleotide sequences and molecular karyotype. Infection, Genetics and Evolution 2, 173-183.

Burgos, J. M., Diez, M., Vigliano, C., Bisio, M., Risso, M., Duffy, T., Cura, C., Brusses, B., Favaloro, L., Leguizamon, M. S., Lucero, R. H., Laguens, R., Levin, M. J., Favaloro, R. and Schijman, A. G. (2010) Molecular identification of Trypanosoma cruzi discrete typing units in end-stage chronic Chagas heart disease and reactivation after heart transplantation. Clinical Infectious Diseases 51, 485-495.

Cardillo, F., de Pinho, R. T., Antas, P. R. and Mengel, J. (2015). Immunity and immune modulation in Trypanosoma cruzi infection. Pathogens and Disease 73, ftv082.

Cardoso, M.S., Reis-Cunha, J. L. and Bartholomeu, D. C. (2016). Evasion of the immune response by Trypanosoma cruzi during acute infection. Frontiers in Immunology 6, 659.

Carod-Artal, F. J. (2010). Trypanosomiasis, cardiomyopathy and the risk of ischemic stroke. Expert Review of Cardiovascular Therapy 8, 717-728. Carod-Artal, F. J. and Gascon, J. (2010). Chagas disease and stroke. Lancet Neurology 9, 533-542.

Chatelain, E. (2016). Chagas disease research and development: is there light at the end of the tunnel? Computational and Structural Biotechnology Fournal 15, 98-103.

Cordova, E., Boschi, A., Ambrosioni, J., Cudos, C. and Corti, M. (2008). Reactivation of Chagas disease with central nervous system involvement in HIV-infected patients in Argentina, 1992-2007. International Fournal of Infectious Diseases 12, 587-592.

Cortes, L. A., Castro, L., Pesce, B., Maya, J. D., Ferreira, J., CastroCastillo, V., Parra, E., Jara, J. A. and López-Muñoz, R. (2015) Novel gallate triphenylphosphonium derivatives with potent antichagasic activity. PLoS ONE 10, e0136852.

Cunha-Neto, E. and Chevillard, C. (2014). Chagas disease cardiomyopathy: immunopathology and genetics. Mediators of Inflammation 2014, 683230 .

Davies, C., Marino Cardozo, R., Sánchez Negrette, O., Mora, M. C., Chung, M. C. and Basombrío, M. A. (2010) Hydroxymethylnitrofurazone is active in a murine model of Chagas' disease. Antimicrobial Agents and Chemotherapy 54, 3584-3589.

Diazgranados, C.A., Saavedra-Trujillo, C.H., Mantilla, M., Valderrama, S. L., Alquichire, C. and Franco-Paredes, C. (2009). Chagasic encephalitis in HIV patients: common presentation of an evolving epidemiological and clinical association. Lancet Infectious Diseases 9, 324-330. DNDi Chagas Disease Fact Sheet (2015). https://www.dndi.org/ wp-content/uploads/2016/10/Factsheet_2015_Chagas_disease.
Eickhoff, C.S., Lawrence, C. T., Sagartz, J. E., Bryant, L. A., Labovitz, A. J., Gala, S. S. and Hoft, D. F. (2010). ECG detection of murine chagasic cardiomyopathy. Fournal of Parasitology 96, 758-764.

Espinoza, B., Rico, T., Sosa, S., Oaxaca, E., Vizcaino-Castillo, A., Caballero, M. L. and Martínez, I. (2010). Mexican Trypanosoma cruzi (TCI) strains with different degrees of virulence induce diverse humoral and cellular immune responses in a murine experimental infection model. Fournal of Biomedicine and Biotechnology 2010, 890672.

Ferraz, M. L., Gazzinelli, R.T., Alves, R. O., Urbina, J.A. and Romanha, A. J. (2007). The anti-Trypanosoma cruzi activity of posaconazole in a murine model of acute Chagas' disease is less dependent on gamma interferon than that of benznidazole. Antimicrobial Agents and Chemotherapy 51, 1359-1364.

Ferreira, A. V., Segatto, M., Menezes, Z., Macedo, A. M., Gelape, C., de Oliveira Andrade, L., Nagajyothi, F., Scherer, P. E., Teixeira, M. M. and Tanowitz, H. B. (2011). Evidence for Trypanosoma cruzi in adipose tissue in human chronic Chagas disease. Microbes and Infection 13, 1002-1005.

Francisco, A. F., Lewis, M. D., Jayawardhana, S., Taylor, M. C., Chatelain, E. and Kelly, J. M. (2015). The limited ability of posaconazole to cure both acute and chronic Trypanosoma cruzi infections revealed by highly sensitive in vivo imaging. Antimicrobial Agents and Chemotherapy 59, 4653-4661.

Francisco, A. F., Jayawardhana, S., Lewis, M. D., White, K. L., Shackleford, D. M., Chen, G., Saunders, J., Osuna-Cabello, M., Read, K. D., Charman, S. A., Chatelain, E. and Kelly, J. M. (2016). Nitroheterocyclic drugs cure experimental Trypanosoma cruzi infections more effectively in the chronic stage than in the acute stage. Scientific Reports 6, 35351.

Franzén, O., Ochaya, S., Sherwood, E., Lewis, M. D., Llewellyn, M. S., Miles, M. A. and Andersson, B. (2011). Shotgun sequencing analysis of trypanosoma cruzi I Sylvio X10/1 and comparison with T. cruzi VI CL Brener. PLoS Neglected Tropical Diseases 5, e984.

Gaspar, L., Moraes, C. B., Freitas-Junior, L. H., Ferrari, S., Costantino, L., Costi, M.P., Coron, R.P., Smith, T. K., SiqueiraNeto, J.L., McKerrow, J.H. and Cordeiro-da-Silva, A. (2015). Current and future chemotherapy for Chagas disease. Current Medicinal Chemistry 22, 4293-4312.

Gaunt, M. W., Yeo, M., Frame, I. A., Stothard, J. R., Carrasco, H. J., Taylor, M. C., Mena, S. S., Veazey, P., Miles, G. A., Acosta, N., de Arias, A. R. and Miles, M. A. (2003). Mechanism of genetic exchange in American trypanosomes. Nature 421, 936-939.

Gironès, N., Cuervo, H. and Fresno, M. (2005) Trypanosoma cruziinduced molecular mimicry and Chagas' disease. Current Topics in Microbiology and Immunology 296, 89-123.

Gruendling, A.P., Massago, M., Teston, A.P., Monteiro, W. M., Kaneshima, E. N., Araújo, S. M., Gomes, M.L., Barbosa, M. and Toledo, M. J. (2015). Impact of benznidazole on infection course in mice experimentally infected with Trypanosoma cruzi I, II, and IV. American Fournal of Tropical Medicine and Hygiene 92, 1178-1189.

Guedes-da-Silva, F. H., Batista, D. G., Meuser, M. B., Demarque, K. C., Fulco, T. O., Araújo, J.S., Da Silva, P. B., Da Silva, C.F., Patrick, D. A., Bakunova, S. M., Bakunov, S. A., Tidwell, R.R., Oliveira, G. M., Britto, C., Moreira, O. C. and Soeiro, M. N. (2016) In vitro and in vivo trypanosomicidal action of novel arylimidamides against Trypanosoma cruzi. Antimicrobial Agents and Chemotherapy 60 $2425-2434$

Gutierrez, F. R., Guedes, P. M., Gazzinelli, R. T. and Silva, J. S. (2009). The role of parasite persistence in pathogenesis of Chagas heart disease. Parasite Immunology 31, 673-685.

Hashimoto, K. and Yoshioka, K. (2012). Review: surveillance of Chagas disease. Advances in Parasitology 79, 375-428.

Huprikar, S., Bosserman, E., Patel, G., Moore, A., Pinney, S., Anyanwu, A., Neofytos, D., Ketterer, D., Striker, R., Silveira, F., Qvarnstrom, Y., Steurer, F., Herwaldt, B. and Montgomery, S. (2013). Donor-derived Trypanosoma cruzi infection in solid organ recipients in the United States, 2001-2011. American fournal of Transplantation 13, 2418-2425.

Iwai, L. K., Juliano, M. A., Juliano, L., Kalil, J. and Cunha-Neto, E. (2005). T-cell molecular mimicry in Chagas disease: identification and partial structural analysis of multiple cross-reactive epitopes between Trypanosoma cruzi B13 and cardiac myosin heavy chain. Fournal of Autoimmunity 24, 111-117.

Katsuno, K., Burrows, J. N., Duncan, K., Hooft van Huijsduijnen, R., Kaneko, T., Kita, K., Mowbray, C. E., Schmatz, D., Warner, P. and Slingsby, B. T. (2015). Hit and lead criteria in drug discovery for infectious diseases of the developing world. Nature Reviews Drug Discovery 14, $751-758$. 
Kessler, R. L., Contreras, V.T., Marliére, N.P., Guarneri, A.A., Villamizar Silva, L. H., Mazzarotto, G. A., Batista, M., Soccol, V. T., Krieger, M. A. and Probst, C. M. (2017). Recently differentiated epimastigotes from Trypanosoma cruzi are infective to the mammalian host. Molecular Microbiology 104, 712-736.

Khare, S., Liu, X., Stinson, M., Rivera, I., Groessl, T., Tuntland, T., Yeh, V., Wen, B., Molteni, V., Glynne, R. and Supek, F. (2015). Antitrypanosomal treatment with benznidazole is superior to posaconazole regimens in mouse models of Chagas disease. Antimicrobial Agents and Chemotherapy 59, 6385-6394.

Kierszenbaum, F. (2005). Where do we stand on the autoimmunity hypothesis of Chagas disease? Trends in Parasitology 21, 513-516.

Kransdorf, E. P., Fishbein, M. C., Czer, L. S., Patel, J. K., Velleca, A., Tazelaar, H. D., Roy, R. R., Steidley, D. E., Kobashigawa, J. A. and Luthringer, D. J. (2016). Pathology of chronic Chagas cardiomyopathy in the United States: A detailed review of 13 cardiectomy cases. American Fournal of Clinical Pathology 146, 191-198.

Kun, H., Moore, A., Mascola, L., Steurer, F., Lawrence, G., Kubak, B., Radhakrishna, S., Leiby, D., Herron, R., Mone, T., Hunter, R. and Kuehnert, M.; Chagas Disease in Transplant Recipients Investigation Team. (2009). Transmission of Trypanosoma cruzi by heart transplantation. Clinical Infectious Diseases 48, 1534-1540. Kurup, S. P. and Tarleton, R. L. (2014) The Trypanosoma cruzi flagellum is discarded via asymmetric cell division following invasion and provides early targets for protective CD8+ T cells. Cell Host and Microbe 16, 439-449. Lages-Silva, E., Crema, E., Ramirez, L. E., Macedo, A. M., Pena, S. D. and Chiari, E. (2001). Relationship between Trypanosoma cruzi and human chagasic megaesophagus: blood and tissue parasitism. American Fournal of Tropical Medicine and Hygiene 65, 435-441.

Lattes, R. and Lasala, M. B. (2014). Chagas disease in the immunosuppressed patient. Clinical Microbiology and Infection 20, 300-309.

Lewis, M. D. and Kelly, J. M. (2016). Putting Trypanosoma cruzi dynamics at the heart of Chagas disease. Trends in Parasitology 32, 899-911 Lewis, M. D., Llewellyn, M. S., Yeo, M., Acosta, N., Gaunt, M. W. and Miles, M. A. (2011). Recent, independent and anthropogenic origins of Trypanosoma cruzi hybrids. PLoS Neglected Tropical Diseases 5, e1363. Lewis, M. D., Fortes Francisco, A., Taylor, M. C., BurrellSaward, H., McLatchie, A. P., Miles, M. A. and Kelly, J. M. (2014) Bioluminescence imaging of chronic Trypanosoma cruzi infections reveals tissue-specific parasite dynamics and heart disease in the absence of locally persistent infection. Cellular Microbiology 16, 1285-1300.

Lewis, M. D., Fortes Francisco, A., Taylor, M. C. and Kelly, J. M. (2015). A new experimental model for assessing drug efficacy against Trypanosoma cruzi infection based on highly sensitive in vivo imaging. fournal of Biomolecular Screening 20, 36-43.

Lewis, M. D., Fortes Francisco, A., Taylor, M. C., Jayawardhana, S. and Kelly, J. M. (2016) Host and parasite genetics shape a link between Trypanosoma cruzi infection dynamics and chronic cardiomyopathy. Cellular Microbiology 18, 1429-1443.

Machado, C. A. and Ayala, F. J. (2001). Nucleotide sequences provide evidence of genetic exchange among distantly related lineages of Trypanosoma cruzi. Proceedings of the National Academy of Sciences of the United States of America 98, 7396-7401.

Marcili, A., Lima, L., Cavazzana, M., Junqueira, A. C., Veludo, H. H., Maia Da Silva, F., Campaner, M., Paiva, F., Nunes, V. L. and Teixeira, M. M. (2009). A new genotype of Trypanosoma cruzi associated with bats evidenced by phylogenetic analyses using SSU rDNA, cytochrome $\mathrm{b}$ and Histone H2B genes and genotyping based on ITS1 rDNA. Parasitology 136, 641-655.

Mejia, A. M., Hall, B.S., Taylor, M.C., Gómez-Palacio, A., Wilkinson, S.R., Triana-Chávez, O. and Kelly, J. M. (2012). Benznidazole-resistance in Trypanosoma cruzi is a readily acquired trait that can arise independently in a single population. Fournal of Infectious Diseases 206, 220-228.

Messenger, L. A., Miles, M. A. and Bern, C. (2015). Between a bug and a hard place: Trypanosoma cruzi genetic diversity and the clinical outcomes of Chagas disease. Expert Review of Anti-infective Therapy 13, 995-1029. Miles, M. A., Llewellyn, M. S., Lewis, M. D., Yeo, M., Baleela, R., Fitzpatrick, S., Gaunt, M. W. and Mauricio, I. L. (2009). The molecular epidemiology and phylogeography of Trypanosoma cruzi and parallel research on Leishmania: looking back and to the future. Parasitology $\mathbf{1 3 6}$ $1509-1528$

Molina, J., Martins-Filho, O., Brener, Z., Romanha, A. J., Loebenberg, D. and Urbina, J. A. (2000). Activities of the triazole derivative SCH 56592 (posaconazole) against drug-resistant strains of the protozoan parasite Trypanosoma (Schizotrypanum) cruzi in immunocompetent and immunosuppressed murine hosts. Antimicrobial Agents and Chemotherapy 44, 150-155.
Molina, I., Gómez i Prat, J., Salvador, F., Treviño, B., Sulleiro, E., Serre, N., Pou, D., Roure, S., Cabezos, J., Valerio, L., BlancoGrau, A., Sánchez-Montalvá, A., Vidal, X. and Pahissa, A. (2014). Randomized trial of posaconazole and benznidazole for chronic Chagas disease. New England Fournal of Medicine 370, 1899-1908.

Molina-Berríos, A., Campos-Estrada, C., Lapier, M., Duaso, J., Kemmerling, U., Galanti, N., Leiva, M., Ferreira, J., LópezMuñoz, R. and Maya, J. D. (2013). Benznidazole prevents endothelial damage in an experimental model of Chagas disease. Acta Tropica 127, 6-13.

Moraes, C. B., Giardini, M. A., Kim, H., Franco, C. H., AraujoJunior, A. M., Schenkman, S., Chatelain, E. and Freitas-Junior, L. H. (2014). Nitroheterocyclic compounds are more efficacious than CYP51 inhibitors against Trypanosoma cruzi: implications for Chagas disease drug discovery and development. Scientific Reports 4, 4703.

Morillo, C. A., Marin-Neto, J. A., Avezum, A., Sosa-Estani, S., Rassi, A., Jr., Rosas, F., Villena, E., Quiroz, R., Bonilla, R., Britto, C., Guhl, F., Velazquez, E., Bonilla, L., Meeks, B., RaoMelacini, P., Pogue, J., Mattos, A., Lazdins, J., Rassi, A., Connolly, S. J. and Yusuf, S. BENEFIT Investigators. (2015). Randomized trial of benznidazole for chronic Chagas' cardiomyopathy. New England Fournal of Medicine 373, 1295-1306.

Morillo, C. A., Waskin, H., Sosa-Estani, S., Del Carmen Bangher, M., Cuneo, C., Milesi, R., Mallagray, M., Apt, W., Beloscar, J., Gascon, J., Molina, I., Echeverria, L. E., Colombo, H., PerezMolina, J. A., Wyss, F., Meeks, B., Bonilla, L. R., Gao, P., Wei, B., McCarthy, M. and Yusuf, S. STOP-CHAGAS Investigators. (2017) Benznidazole and posaconazole in eliminating parasites in asymptomatic T. cruzi carriers: the STOP-CHAGAS trial. Fournal of the American College of Cardiology 69, 939-947.

Olivieri, B. P., Molina, J. T., de Castro, S. L., Pereira, M. C., Calvet, C. M., Urbina, J. A. and Araújo-Jorge, T. C. (2010). A comparative study of posaconazole and benznidazole in the prevention of heart damage and promotion of trypanocidal immune response in a murine model of Chagas disease. International Fournal of Antimicrobial Agents 36, 79-83.

Oliveira, M. T., Branquinho, R. T., Alessio, G. D., Mello, C. G., Nogueira-de-Paiva, N. C., Carneiro, C. M., Toledo, M. J., Reis, A. B., Martins-Filho, O. A. and Lana, M. (2017). Tci, TcII and TcVI Trypanosoma cruzi samples from Chagas disease patients with distinct clinical forms and critical analysis of in vitro and in vivo behavior, response to treatment and infection evolution in murine model. Acta Tropica 167, 108-120.

Pérez-Molina, J. A., Norman, F. and López-Vélez, R. (2012). Chagas disease in non-endemic countries: epidemiology, clinical presentation and treatment. Current Infectious Disease Reports 14, 263-274.

Perin, L., Moreira da Silva, R., da Silva Fonseca, K., Mirelle de Oliveira Cardoso, J., Mathias, F. A., Reis, L. E., Molina, I., CorreaOliveira, R., de Abreu Vieira, P. M. and Carneiro, C. M. (2017). Pharmacokinetic and tissue distribution of benznidazole after oral administration in mice. Antimicrobial Agents and Chemotherapy 61, e02410e02416.

Pinto, C. M., Kalko, E. K., Cottontail, I., Wellinghausen, N. and Cottontail, V. M. (2012). Tcbat a bat-exclusive lineage of Trypanosoma cruzi in the Panama Canal Zone, with comments on its classification and the use of the $18 \mathrm{~S}$ rRNA gene for lineage identification. Infection, Genetics and Evolution 12, 1328-1332.

Postan, M., Bailey, J. J., Dvorak, J. A., McDaniel, J. P. and Pottala, E. W. (1987). Studies of Trypanosoma cruzi clones in inbred mice. III. Histopathological and electrocardiographical responses to chronic infection. American fournal of Tropical Medicine and Hygiene 37, 541-549. Requena-Méndez, A., Aldasoro, E., de Lazzari, E., Sicuri, E., Brown, M., Moore, D.A., Gascon, J. and Muñoz, J. (2015). Prevalence of Chagas disease in Latin-American migrants living in Europe: a systematic review and meta-analysis. PLoS Neglected Tropical Diseases $\mathbf{9}$, $\mathrm{e} 0003540$

Ribeiro, A. L., Nunes, M.P., Teixeira, M. M. and Rocha, M. O. (2012). Diagnosis and management of Chagas disease and cardiomyopathy. Nature Reviews Cardiology 9, 576-589.

Rodriguez, H. O., Guerrero, N. A., Fortes, A., Santi-Rocca, J., Gironès, N. and Fresno, M. (2014). Trypanosoma cruzi strains cause different myocarditis patterns in infected mice. Acta Tropica 139, 57-66. Rossi, M. A., Ramos, S. G. and Bestetti, R. B. (2003). Chagas' heart disease: clinical-pathological correlation. Frontiers in Bioscience 8, e94-109. Santos, F. M., Mazzeti, A. L., Caldas, S., Gonçalves, K. R., Lima, W. G., Torres, R. M. and Bahia, M. T. (2016). Chagas cardiomyopathy: the potential effect of benznidazole treatment on diastolic dysfunction and cardiac damage in dogs chronically infected with Trypanosoma cruzi. Acta Tropica $161,44-54$ 
Sbaraglini, M. L., Bellera, C. L., Fraccaroli, L., Larocca, L., Carrillo, C., Talevi, A. and Alba Soto, C. D. (2016). Novel cruzipain inhibitors for the chemotherapy of chronic Chagas disease. International Fournal of Antimicrobial Agents 48, 91-95.

Schijman, A. G., Vigliano, C. A., Viotti, R. J., Burgos, J. M., Brandariz, S., Lococo, B.E., Leze, M. I., Armenti, H. A. and Levin, M. J. (2004). Trypanosoma cruzi DNA in cardiac lesions of Argentinean patients with end-stage chronic chagas heart disease. American Fournal of Tropical Medicine and Hygiene 70, 210-220. Schijman, A. G., Bisio, M., Orellana, L., Sued, M., Duffy, T., Mejia Jaramillo, A. M., Cura, C., Auter, F., Veron, V., Qvarnstrom, Y., Deborggraeve, S., Hijar, G., Zulantay, I., Lucero, R. H., Velazquez, E., Tellez, T., Sanchez Leon, Z., Galvão, L., Nolder, D., Monje Rumi, M., Levi, J. E., Ramirez, J. D., Zorrilla, P., Flores, M., Jercic, M.I., Crisante, G., Añez, N., De Castro, A. M., Gonzalez, C. I., Acosta Viana, K., et al. (2011). International study to evaluate PCR methods for detection of trypanosoma cruzi DNA in blood samples from Chagas disease patients. PLoS Neglected Tropical Diseases 5, e931.

Schlemper, B. R., Jr., Avila, C. M., Coura, J. R. and Brener, Z. (1983). Course of infection and histopathological lesions in mice infected with seventeen Trypanosoma cruzi strains isolated from chronic patients. Revista da Sociedade Brasileira de Medicina Tropical, 16, 23-30.

Tanowitz, H. B., Scherer, P. E., Mota, M. M. and Figueiredo, L. M. (2017). Adipose tissue: a safe haven for parasites? Trends parasitol. Trends in Parasitology 33, 276-284.

Tarleton, R. L. (2015). CD8+ t cells in Trypanosoma cruzi infection. Seminars in Immunopathology 37, 233-238.

Taylor, M. C., Lewis, M. D., Fortes Francisco, A., Wilkinson, S. R. and Kelly, J. M. (2015). The Trypanosoma cruzi vitamin C dependent peroxidase confers protection against oxidative stress but is not a determinant of virulence. PLoS Neglected Tropical Diseases 9, e0003707.

Teixeira, A. R., Hecht, M.M., Guimaro, M. C., Sousa, A. O. and Nitz, N. (2011). Trypanosoma cruzi in the chicken model: Chagas-like heart disease in the absence of parasitism. Clinical Microbiology Reviews 24, 592-630.

Teston, A.P., Monteiro, W. M., Reis, D., Bossolani, G. D. Gomes, M. L., de Araújo, S. M., Bahia, M. T., Barbosa, M. G. and Toledo, M. J. (2013). In vivo susceptibility to benznidazole of Trypanosoma cruzi strains from the western Brazilian Amazon. Tropical Medicine and International Health 18, 85-95.

Toledo, M. J., Bahia, M. T., Carneiro, C. M., Martins-Filho, O. A., Tibayrenc, M., Barnabé, C., Tafuri, W. L. and de Lana, M. (2003) Chemotherapy with benznidazole and itraconazole for mice infected with different Trypanosoma cruzi clonal genotypes. Antimicrobial Agents and Chemotherapy 47, 223-230.
Trindade, S., Rijo-Ferreira, F., Carvalho, T., Pinto-Neves, D., Guegan, F., Aresta-Branco, F., Bento, F., Young, S. A., Pinto, A., Van Den Abbeele, J., Ribeiro, R.M., Dias, S., Smith, T. K. and Figueiredo, L. M. (2016). Trypanosoma brucei parasites occupy and functionally adapt to the adipose tissue in mice. Cell Host and Microbe 19, 837-848.

Tyler, K. M. and Engman, D. M. (2001). The life cycle of Trypanosoma cruzi revisited. International Fournal for Parasitology 31, 472-481.

Vago, A. R., Macedo, A.M., Adad, S. J., Reis, D. D. and CorrêaOliveira, R. (1996). PCR detection of Trypanosoma cruzi DNA in oesophageal tissues of patients with chronic digestive Chagas' disease. Lancet 348, 91-92.

Villar, J. C., Perez, J. G., Cortes, O. L., Riarte, A., Pepper, M., MarinNeto, J. A. and Guyatt, G. H. (2014). Trypanocidal drugs for chronic asymptomatic Trypanosoma cruzi infection. The Cochrane Database of Systematic Reviews 5, CD003463.

Villarreal, D., Barnabé, C., Sereno, D. and Tibayrenc, M. (2004) Lack of correlation between in vitro susceptibility to benznidazole and phylogenetic diversity of Trypanosoma cruzi, the agent of Chagas disease. Experimental Parasitology 108, 24-31.

Vitelli-Avelar, D. M., Sathler-Avelar, R., Mattoso-Barbosa, A. M. Gouin, N., Perdigão-de-Oliveira, M., Valério-Dos-Reis, L. Costa, R.P., Elói-Santos, S. M., Gomes, M. S., Amaral, L. R., Teixeira-Carvalho, A., Martins-Filho, O. A., Dick, E. J., Jr., Hubbard, G. B., VandeBerg, J.F. and VandeBerg, J. L. (2017). Cynomolgus macaques naturally infected with Trypanosoma cruzi-I exhibit an overall mixed pro-inflammatory/modulated cytokine signature characteristic of human Chagas disease. PLoS Neglected Tropical Diseases 11, e0005233.

Wilkinson, S. R. and Kelly, J. M. (2009). Trypanocidal drugs: mechanisms, resistance and new targets. Expert Reviews in Molecular Medicine 11, e31, pp1-24.

Wilkinson, S. R., Taylor, M. C., Horn, D., Kelly, J.M. and Cheeseman, I. (2008). A mechanism for cross-resistance to nifurtimox and benznidazole in trypanosomes. Proceedings of the National Academy of Sciences of the United States of America 105, 5022-5027.

Yasukawa, K., Patel, S. M., Flash, C. A., Stager, C. E., Goodman, J. C. and Woc-Colburn, L. (2014). Trypanosoma cruzi meningoencephalitis in a patient with acquired immunodeficiency syndrome. American fournal of Tropical Medicine and Hygiene 91, 84-85.

Zingales, B., Miles, M. A., Campbell, D.A., Tibayrenc, M., Macedo, A. M., Teixeira, M. M., Schijman, A. G., Llewellyn, M. S. Lages-Silva, E., Machado, C. R., Andrade, S. G. and Sturm, N. R. (2012). The revised Trypanosoma cruzi subspecific nomenclature: rationale, epidemiological relevance and research applications. Infection, Genetics and Evolution 12, 240-253. 